\title{
Iterative Algorithms for New General Systems of Set-Valued Variational Inclusions Involving $(A, \eta)$-Maximal Relaxed Monotone Operators
}

\author{
Ting-jian Xiong ${ }^{1}$ and Heng-you Lan ${ }^{1,2}$ \\ ${ }^{1}$ Department of Mathematics, Sichuan University of Science \& Engineering, Zigong, Sichuan 643000, China \\ ${ }^{2}$ Artificial Intelligence Key Laboratory of Sichuan Province, Zigong, Sichuan 643000, China \\ Correspondence should be addressed to Heng-you Lan; hengyoulan@163.com
}

Received 24 March 2014; Accepted 16 May 2014; Published 5 June 2014

Academic Editor: Jian-Wen Peng

Copyright (c) 2014 T.-j. Xiong and H.-y. Lan. This is an open access article distributed under the Creative Commons Attribution License, which permits unrestricted use, distribution, and reproduction in any medium, provided the original work is properly cited.

We introduce and study a class of new general systems of set-valued variational inclusions involving $(A, \eta)$-maximal relaxed monotone operators in Hilbert spaces. By using the general resolvent operator technique associated with $(A, \eta)$-maximal relaxed monotone operators, we construct some new iterative algorithms for finding approximation solutions to the general system of setvalued variational inclusion problem and prove the convergence of this algorithm. Our results improve and extend some known results.

\section{Introduction}

It is well known that variational inequalities and variational inclusions, which have been extended and generalized in different directions by using novel and innovative techniques and ideas, provide mathematical models to some problems arising in economics, mechanics, engineering science, and other pure and applied sciences. Among these methods, the resolvent operator technique is very important. See, for example, [1-17] and the references therein.

Recently, Huang and Fang [18] introduced a system of order complementarity problems and established some existence results for the system using fixed-point theory. Verma [19] introduced and studied some systems of the system variational inequalities and developed some iterative algorithms for approximating the solutions of the systems of variational inequalities. Cho et al. [20] introduced and studied a new system of nonlinear variational inequalities in Hilbert spaces. The authors also proved some existence and uniqueness theorems of solutions for the system and also constructed an iterative algorithm for approximating the solution of the system of nonlinear variational inequalities. Further, Fang et al. [1], Yan et al. [2], Fang and Huang [3], and Cao [4] considered some new systems of variational inclusions involving $H$-monotone operators and $(H, \eta)$ monotone operators in Hilbert space, respectively. Using the corresponding resolvent operator associated with $\mathrm{H}$ monotone operators and $(H, \eta)$-monotone operators, the authors proved the existence of solutions for these new systems of variational inclusions and constructed a new algorithm for approximating the solution of this system and discussed the convergence of the sequence of iterations generated by the algorithm.

Very recently, Lan et al. [5] and Peng and Zhao [7] introduced and studied a new system of nonlinear $A$-monotone multivalued variational inclusions in Hilbert spaces, respectively. By using the concept and properties of $A$-monotone operators and the resolvent operator technique associated with $A$-monotone operators due to Verma [8], the author constructed a new iterative algorithm for solving this system of nonlinear multivalued variational inclusions associated with $A$-monotone operators in Hilbert spaces and proved the existence of solutions for the nonlinear multivalued variational inclusion systems and the convergence of iterative sequences generated by the algorithm. For more details, see, for example, [1-5, 7, 8, 10-19, 21-25]. 
On the other hand, Lan [6] first introduced a new concept of $(A, \eta)$-monotone (the so-called $(A, \eta)$-maximal relaxed monotone [9]) operators, which generalizes the $(H, \eta)$ monotonicity, $A$-monotonicity, and other existing monotone operators as special cases, and studied some properties of $(A, \eta)$-monotone operators and defined resolvent operators associated with $(A, \eta)$-monotone operators.

Inspired and motivated by the above works, the purpose of this paper is to consider the following new general system of set-valued variational inclusions involving relative $(A, \eta)$-maximal monotone operators in Hilbert spaces: find $\left(x_{1}^{*}, x_{2}^{*}, \ldots, x_{m}^{*}\right) \in H_{1} \times H_{2} \times \cdots \times H_{m}$ and $u_{i j} \in U_{i j}\left(x_{j}^{*}\right)$ such that

$$
0 \in F_{i}\left(u_{i 1}, \ldots, u_{i i-1}, x_{i}^{*}, u_{i i+1}, \ldots, u_{i m}\right)+M_{i}\left(x_{i}^{*}\right),
$$

where $m$ is a given positive integer, $F_{i}: H_{1} \times H_{2} \times \cdots \times H_{m} \rightarrow$ $H_{i}, A_{i}: H_{i} \rightarrow H_{i}$, and $\eta_{i}: H_{i} \times H_{i} \rightarrow H_{i}$ are singlevalued operators, $U_{i j}: H_{j} \rightarrow 2^{H_{j}}$ is a set-valued operator, $M_{i}: H_{i} \rightarrow 2^{H_{i}}$ is $\left(A_{i}, \eta_{i}\right)$-maximal relaxed monotone, and $i, j=1,2, \ldots, m$ and $i \neq j$.

Some special cases of the problem (1) had been studied by many authors. Here, we mention some of them as follows.

Case 1. If $m=2$, then the problem (1) reduces to the problem of finding $\left(x_{1}^{*}, x_{2}^{*}\right) \in H_{1} \times H_{2}$ and $u_{1} \in U_{1}\left(x_{1}^{*}\right)$ and $u_{2} \in$ $U_{2}\left(x_{2}^{*}\right)$ such that

$$
\begin{aligned}
& 0 \in F_{1}\left(x_{1}^{*}, u_{2}\right)+M_{1}\left(x_{1}^{*}\right), \\
& 0 \in F_{2}\left(u_{1}, x_{2}^{*}\right)+M_{2}\left(x_{2}^{*}\right) .
\end{aligned}
$$

The problem (2) is called a nonlinear set-valued variational inclusion system problem, which was considered and studied by Agarwal and Verma [9].

Case 2. When $m=2$ and $M_{i}\left(x_{i}\right)=\partial \varphi_{i}\left(x_{i}\right)$, for all $x_{i} \in H_{i}$, $i=1,2$, where $\varphi_{i}: H_{i} \rightarrow R \cup\{+\infty\}$ is proper, convex, and lower semicontinuous functional and $\partial \varphi_{i}$ denotes the subdifferential operator of $\varphi_{i}$, then problem (1) becomes the following system of set-valued mixed variational inequalities: find $\left(x_{1}^{*}, x_{2}^{*}\right) \in H_{1} \times H_{2}$ and $u_{1} \in U_{1}\left(x_{1}^{*}\right)$ and $u_{2} \in U_{2}\left(x_{2}^{*}\right)$ such that

$$
\begin{array}{ll}
\left\langle F_{1}\left(x_{1}^{*}, u_{2}\right), x-x_{1}^{*}\right\rangle+\varphi_{1}(x)-\varphi_{1}\left(x_{1}^{*}\right) \geq 0, & \forall x \in H_{1}, \\
\left\langle F_{2}\left(u_{1}, x_{2}^{*}\right), y-x_{2}^{*}\right\rangle+\varphi_{2}(y)-\varphi_{2}\left(x_{2}^{*}\right) \geq 0, \quad \forall y \in H_{2} .
\end{array}
$$

If $U_{1}=U_{2} \equiv I$, the identity operator, then the problem (3) reduces to the following problem of finding $\left(x_{1}^{*}, x_{2}^{*}\right) \in H_{1} \times$ $\mathrm{H}_{2}$ such that

$$
\begin{aligned}
& \left\langle F_{1}\left(x_{1}^{*}, x_{2}^{*}\right), x-x_{1}^{*}\right\rangle+\varphi_{1}(x)-\varphi_{1}\left(x_{1}^{*}\right) \geq 0, \quad \forall x \in H_{1}, \\
& \left\langle F_{2}\left(x_{1}^{*}, x_{2}^{*}\right), y-x_{2}^{*}\right\rangle+\varphi_{2}(y)-\varphi_{2}\left(x_{2}^{*}\right) \geq 0, \quad \forall y \in H_{2},
\end{aligned}
$$

which is called the system of nonlinear variational inequalities considered by Cho et al. [20]. Some specializations of problem (4) are dealt by Kim and Kim [21].
Case 3. If $m=2$ and $U_{1}=U_{2} \equiv I$, then the problem (1) reduces to finding $\left(x_{1}^{*}, x_{2}^{*}\right) \in H_{1} \times H_{2}$ such that

$$
\begin{aligned}
& 0 \in F_{1}\left(x_{1}^{*}, x_{2}^{*}\right)+M_{1}\left(x_{1}^{*}\right), \\
& 0 \in F_{2}\left(x_{1}^{*}, x_{2}^{*}\right)+M_{2}\left(x_{2}^{*}\right),
\end{aligned}
$$

which was considered by Fang et al. [1].

In brief, the problem (1) is the most general and unifying system form, so long as, for appropriate and suitable choices of positive integer $m$ and operators $F_{i}, A_{i}, \eta_{i}, M_{i}$, and $U_{i j}$ for $i, j=1,2, \ldots, m$, one can know that the problem (1) includes a number of known general problems of variational character, including variational inequality (system) problems and variational inclusion (system) problems as special cases. For more details, see $[1-5,7-25]$ and the reference therein.

Furthermore, in this paper, we will construct some new iterative algorithms to approximate the solution of the general system of set-valued variational inclusions and prove the convergence of the sequences generated by the algorithms in Hilbert spaces.

\section{Preliminaries}

Thereafter, let $H, H_{i}(i=1,2, \ldots, m)$ be real Hilbert spaces endowed with the norm $\|\cdot\|$ and inner product $\langle\cdot, \cdot\rangle$. Let $2^{H}$ and $C(H)$ denote the family of all the nonempty subsets of $H$ and the family of all closed subsets of $H$, respectively.

In order to get the main results of the paper, we need the following concepts and lemmas.

Definition 1. Let $A: H \rightarrow H$ be a single-valued operator. Then the map $A$ is said to be

(i) $\alpha$-strongly monotone, if there exists a constant $\alpha>0$ such that

$$
\langle A(x)-A(y), x-y\rangle \geq \alpha\|x-y\|^{2}, \quad \forall x, y \in H
$$

(ii) $\beta$-Lipschitz continuous, if there exists a constant $\beta>$ 0 such that

$$
\|A x-A y\| \leq \beta\|x-y\|, \quad \forall x, y \in H .
$$

Definition 2. Let $\eta: H \times H \rightarrow H$ and $A: H \rightarrow H$ be singlevalued operators; let $M: H \rightarrow 2^{H}$ be set-valued operator. Then

(i) $\eta$ is said to be $\tau$-Lipschitz continuous, if there exists a constant $\tau>0$ such that

$$
\|\eta(x, y)\| \leq \tau\|x-y\|, \quad \forall x, y \in H ;
$$

(ii) $A$ is said to be $\eta$-monotone, if

$$
\langle A(x)-A(y), \eta(x, y)\rangle \geq 0, \quad \forall x, y \in H ;
$$

(iii) $A$ is said to be strictly $\eta$-monotone, if $A$ is $\eta$-monotone and

$$
\langle A(x)-A(y), \eta(x, y)\rangle=0 \quad \text { iff } x=y ;
$$


(iv) $A$ is said to be $(r, \eta)$-strongly monotone, if there exists a constant $r>0$ such that

$$
\langle A(x)-A(y), \eta(x, y)\rangle \geq r\|x-y\|^{2}, \quad \forall x, y \in H ;
$$

(v) $M$ is said to be $(m, \eta)$-relaxed monotone, if there exists a constant $m>0$ such that

$$
\begin{array}{r}
\langle u-v, \eta(x, y)\rangle \geq(-m)\|x-y\|^{2}, \quad \forall x, y \in H, \\
u \in M(x), \quad v \in M(y) ;
\end{array}
$$

(vi) $M$ is said to be $(A, \eta)$-maximal relaxed monotone, if $M$ is $(m, \eta)$-relaxed monotone and

$$
(A+\lambda M)(H)=H, \quad \forall \lambda>0 .
$$

Definition 3. For $i=1,2, \ldots, m$, let $H_{i}$ be a Hilbert space and let $A_{i}: H_{i} \rightarrow H_{i}$ be a single-valued operator; then nonlinear operator $F_{i}: H_{1} \times H_{2} \times \cdots \times H_{m} \rightarrow H_{i}$ is said to be

(i) $\mu_{i}$-strongly monotone with respect to $A_{i}$ in the $i$ th argument, if there exist constants $\mu_{i}>0$ such that, for $x_{i}^{1}, x_{i}^{2} \in H_{i}, i=1,2, \ldots, m$,

$$
\begin{aligned}
& \left\langle F_{i}\left(\ldots, x_{i}^{1}, \ldots\right)-F_{i}\left(\ldots, x_{i}^{2}, \ldots\right), A_{i}\left(x_{i}^{1}\right)-A_{i}\left(x_{i}^{2}\right)\right\rangle \\
& \quad \geq \mu_{i}\left\|x_{i}^{1}-x_{i}^{2}\right\|^{2} ;
\end{aligned}
$$

(ii) $\left(c_{i}, \mu_{i}\right)$-relaxed cocoercive with respect to $A_{i}$ (or relative $\left(c_{i}, \mu_{i}\right)$-relaxed cocoercive) in the $i$ th argument, if there exist constants $c_{i}, \mu_{i}>0$ such that, for $x_{i}^{1}, x_{i}^{2} \in$ $H_{i}, i=1,2, \ldots, m$,

$$
\begin{aligned}
& \left\langle F_{i}\left(\ldots, x_{i}^{1}, \ldots\right)-F_{i}\left(\ldots, x_{i}^{2}, \ldots\right), A_{i}\left(x_{i}^{1}\right)-A_{i}\left(x_{i}^{2}\right)\right\rangle \\
& \geq\left(-c_{i}\right)\left\|F_{i}\left(\ldots, x_{i}^{1}, \ldots\right)-F_{i}\left(\ldots, x_{i}^{2}, \ldots\right)\right\|^{2}+\mu_{i}\left\|x_{i}^{1}-x_{i}^{2}\right\|^{2} ;
\end{aligned}
$$

(iii) $\zeta_{i j}$-Lipschitz continuous in the $j$ th argument, if there exists constant $\zeta_{i j}>0$ such that, for $x_{j}, y_{j} \in H_{j}, j=$ $1,2, \ldots, m$,

$$
\begin{aligned}
& \| F_{i}\left(x_{1}, \ldots, x_{j-1}, x_{j}, x_{j+1}, \ldots, x_{m}\right) \\
& -F_{i}\left(x_{1}, \ldots, x_{j-1}, y_{j}, x_{j+1}, \ldots, x_{m}\right) \| \\
& \quad \leq \zeta_{i j}\left\|x_{j}-y_{j}\right\| .
\end{aligned}
$$

Remark 4. When $m=1$, then Definition 3 reduces to the corresponding concept of the relative strong monotonicity, relative relaxed cocoercive, and Lipschitz continuity.

Definition 5. Let $\eta: H \times H \rightarrow H$ be a single-valued operator, let $A: H \rightarrow H$ be a strictly $\eta$-monotone operator, and let $M$ : $H \rightarrow 2^{H}$ be an $(A, \eta)$-maximal relaxed monotone operator. Then general resolvent operator $R_{M, \lambda}^{A, \eta}: H \rightarrow H$ is defined by

$$
R_{M, \lambda}^{A, \eta}(z)=(A+\lambda M)^{-1}(z), \quad \forall z \in H,
$$

where $\lambda>0$ is a constant.
Lemma 6 (see [6]). Let $\eta: H \times H \rightarrow H$ be a $\tau$-Lipschitz continuous operator, let $A: H \rightarrow H$ be an $(r, \eta)$-strongly monotone operator, and let $M: H \rightarrow 2^{H}$ be an $(A, \eta)$ maximal relaxed monotone operator. Then general resolvent operator $R_{M, \lambda}^{A, \eta}: H \rightarrow H$ is $\tau /(r-\lambda m)$-Lipschitz continuous; that is,

$$
\left\|R_{M, \lambda}^{A, \eta}(x)-R_{M, \lambda}^{A, \eta}(y)\right\| \leq \frac{\tau}{r-\lambda m}\|x-y\|, \quad \forall x, y \in H,
$$

where $-\lambda m>0$.

Next, we define the Hausdorff pseudometric $D: C(H) \times$ $C(H) \rightarrow \mathbb{R} \cup\{+\infty\}$ as follows:

$$
\begin{array}{r}
D(U, V)=\max \left\{\sup _{x \in U} \inf _{y \in V}\|x-y\|, \sup _{y \in V} \inf _{x \in U}\|x-y\|\right\}, \\
\forall U, V \in C(H) .
\end{array}
$$

Note that if $C(H)$ is restricted to closed bounded subsets of the family $C B(H)$, then the Hausdorff pseudometric $D$ reduces to Hausdorff metric $\widehat{H}: C B(H) \times C B(H) \rightarrow \mathbb{R}$ defined by

$$
\begin{array}{r}
\widehat{H}(U, V)=\max \left\{\sup _{x \in U} \inf _{y \in V}\|x-y\|, \sup _{y \in V} \inf _{x \in U}\|x-y\|\right\}, \\
\forall U, V \in C B(H) .
\end{array}
$$

Definition 7. A set-valued operator $U: H \rightarrow 2^{H}$ is said to be $D$ - $\gamma$-Lipschitz continuous if there exists a constant $\gamma>0$ such that

$$
D(U(x), U(y)) \leq \gamma\|x-y\|, \quad \forall x, y \in H .
$$

Lemma 8. Let $\theta$ be a constant and $0<\omega<1$; then function $f(\omega)=1-\omega+\omega \theta$, for $\omega \in[0,1]$, is nonnegative and strictly decreases and $f(\omega) \in[0,1]$. Further, if $\omega \neq 0$, then $f(\omega) \in$ $(0,1)$.

Proof. Since $f(\omega)$ is linear function, the conclusions immediately hold.

\section{Iterative Algorithm and Convergence}

In this section, we first prove the equivalence between the problem (1) and the problem of finding the fixed points of the general resolvent operator $R_{M, \lambda}^{A, \eta}$ associated with $(A, \eta)$ maximal relaxed monotone operators. This equivalence is quite general and very important from a numerical point of view. Then, by using the equivalence, some new iterative algorithms for finding the approximation solutions of the problem (1) are analyzed. Further, the convergence criteria for the algorithms are also discussed.

Lemma 9. Let $\left(x_{1}^{*}, x_{2}^{*}, \ldots, x_{m}^{*}\right) \in H_{1} \times H_{2} \times \cdots \times$ $H_{m}$ and $u_{i j} \in U_{i j}\left(x_{j}^{*}\right)(i, j=1,2, \ldots, m, j \neq i)$; then 
$\left(x_{1}^{*}, x_{2}^{*}, \ldots, x_{m}^{*}, u_{12}, \ldots, u_{1 m}, \ldots, u_{m 1}, \ldots, u_{m m-1}\right)$ (denoted by $(*))$ is a solution of the problem (1) if and only if (*) satisfy

$x_{i}^{*}=R_{M_{i}, \rho_{i}}^{A_{i}, \eta_{i}}\left[A_{i}\left(x_{i}^{*}\right)-\rho_{i} F_{i}\left(u_{i 1}, \ldots, u_{i i-1}, x_{i}^{*}, u_{i i+1}, \ldots, u_{i m}\right)\right]$,

where $R_{M_{i}, \rho_{i}}^{A_{i}, \eta_{i}}=\left(A_{i}+\rho_{i} M_{i}\right)^{-1}$ and $\rho_{i}>0$ is a constant, for $i=1,2, \cdots, m$.

Proof. Let $(*)$ satisfy the relation (22). By Definition 5 of general resolvent operator, the equality (22) holds if and only if

$$
\begin{aligned}
& A_{i}\left(x_{i}^{*}\right)-\rho_{i} F_{i}\left(u_{i 1}, \ldots, u_{i i-1}, x_{i}^{*}, u_{i i+1}, \ldots, u_{i m}\right) \\
& \quad \in\left(A_{i}+\rho_{i} M_{i}\right)\left(x_{i}^{*}\right)
\end{aligned}
$$

that is,

$$
0 \in F_{i}\left(u_{i 1}, \ldots, u_{i i-1}, x_{i}^{*}, u_{i i+1}, \ldots, u_{i m}\right)+M_{i}\left(x_{i}^{*}\right)
$$

where $i=1,2, \ldots, m$. Hence $(*)$ are the solution of the problem (1). This completes the proof.

By using formula (22) and Nadler [26], we can develop the following new iterative algorithms.

Algorithm 10. Consider the following.

Step 1. Choose $\left(x_{1}^{0}, x_{2}^{0}, \ldots, x_{m}^{0}\right) \in H_{1} \times H_{2} \times \cdots \times H_{m}$ and $u_{i j}^{0} \in U_{i j}\left(x_{j}^{0}\right)$ for $i, j=1,2, \ldots, m, j \neq i$.

Step 2. Let

$$
\begin{aligned}
x_{i}^{n+1}= & \left(1-\lambda_{n}-\delta_{n}\right) x_{i}^{n} \\
& +\lambda_{n} R_{M_{i}, \rho_{i}}^{A_{i}, \eta_{i}}\left[A_{i}\left(x_{i}^{n}\right)\right. \\
& \left.\quad-\rho_{i} F_{i}\left(u_{i 1}^{n}, \ldots, u_{i i-1}^{n}, x_{i}^{n}, u_{i i+1}^{n}, \ldots, u_{i m}^{n}\right)\right],
\end{aligned}
$$

for all $i=1,2, \ldots, m$ and $n=0,1,2, \ldots$, where $\lambda_{n}$ and $\delta_{n}$ are nonnegative constants such that $0<\lambda_{n}+\delta_{n} \leq 1$ and $\lim \inf _{n \geq 0} \lambda_{n}>0$.

Step 3. Choose $u_{i j}^{n+1} \in U_{i j}\left(x_{j}^{n+1}\right)(i, j=1,2, \ldots, m, j \neq i)$ such that

$$
\left\|u_{i j}^{n+1}-u_{i j}^{n}\right\| \leq\left(1+\frac{1}{n+1}\right) D_{j}\left(U_{i j}\left(x_{j}^{n+1}\right), U_{i j}\left(x_{j}^{n}\right)\right)
$$

where $D_{j}(\cdot, \cdot)$ is the Hausdorff pseudometric on $C\left(H_{j}\right)$.

Step 4. If $x_{i}^{n+1}$ and $u_{i j}^{n+1}(i, j=1,2, \ldots, m)$ satisfy (25) to sufficient accuracy, stop. Otherwise, set $n:=n+1$ and return to Step 2.
Algorithm 11. Consider the following.

Step 1. Choose $\left(x_{1}^{0}, x_{2}^{0}, \ldots, x_{m}^{0}\right) \in H_{1} \times H_{2} \times \cdots \times H_{m}$ and $u_{i j}^{0} \in U_{i j}\left(x_{j}^{0}\right)$, for $i, j=1,2, \ldots, m, j \neq i$.

Step 2. Let

$$
\begin{aligned}
x_{i}^{n+1}=(1-\lambda-\delta) & x_{i}^{n} \\
& +\lambda R_{M_{i}, \rho_{i}}^{A_{i}, \eta_{i}}\left[A_{i}\left(x_{i}^{n}\right)\right. \\
& \left.\quad-\rho_{i} F_{i}\left(u_{i 1}^{n}, \ldots, u_{i i-1}^{n}, x_{i}^{n}, u_{i i+1}^{n}, \ldots, u_{i m}^{n}\right)\right],
\end{aligned}
$$

for all $i=1,2, \ldots, m$ and $n=0,1,2, \ldots$, where $\lambda$ and $\delta$ are nonnegative constants such that $0<\lambda+\delta \leq 1$.

Step 3. Choose $u_{i j}^{n+1} \in U_{i j}\left(x_{j}^{n+1}\right)(i, j=1,2, \ldots, m, j \neq i)$ such that

$$
\left\|u_{i j}^{n+1}-u_{i j}^{n}\right\| \leq\left(1+\frac{1}{n+1}\right) D_{j}\left(U_{i j}\left(x_{j}^{n+1}\right), U_{i j}\left(x_{j}^{n}\right)\right),
$$

where $D_{j}(\cdot, \cdot)$ is the Hausdorff pseudometric on $C\left(H_{j}\right)$.

Step 4. If $x_{i}^{n+1}$ and $u_{i j}^{n+1}(i, j=1,2, \ldots, m)$ satisfy (27) to sufficient accuracy, stop. Otherwise, set $n:=n+1$ and return to Step 2.

Algorithm 12. Consider the following.

Step 1. Choose $\left(x_{1}^{0}, x_{2}^{0}, \ldots, x_{m}^{0}\right) \in H_{1} \times H_{2} \times \cdots \times H_{m}$ and $u_{i j}^{0} \in U_{i j}\left(x_{j}^{0}\right)$, for $i, j=1,2, \ldots, m, j \neq i$.

Step 2. Let

$$
\begin{aligned}
x_{i}^{n+1}= & (1-\lambda) x_{i}^{n} \\
& +\lambda R_{M_{i}, \rho_{i}}^{A_{i}, \eta_{i}}\left[A_{i}\left(x_{i}^{n}\right)\right. \\
& \left.\quad-\rho_{i} F_{i}\left(u_{i 1}^{n}, \ldots, u_{i i-1}^{n}, x_{i}^{n}, u_{i i+1}^{n}, \ldots, u_{i m}^{n}\right)\right],
\end{aligned}
$$

for all $i=1,2, \ldots, m$ and $n=0,1,2, \ldots$, where $\lambda$ is a nonnegative constant such that $0<\lambda \leq 1$.

Step 3. Choose $u_{i j}^{n+1} \in U_{i j}\left(x_{j}^{n+1}\right)(i, j=1,2, \ldots, m, j \neq i)$ such that

$$
\left\|u_{i j}^{n+1}-u_{i j}^{n}\right\| \leq\left(1+\frac{1}{n+1}\right) D_{j}\left(U_{i j}\left(x_{j}^{n+1}\right), U_{i j}\left(x_{j}^{n}\right)\right),
$$

where $D_{j}(\cdot, \cdot)$ is the Hausdorff pseudometric on $C\left(H_{j}\right)$.

Step 4. If $x_{i}^{n+1}$ and $u_{i j}^{n+1}(i, j=1,2, \ldots, m)$ satisfy (29) to sufficient accuracy, stop. Otherwise, set $n:=n+1$ and return to Step 2.

Remark 13. Let $m=2$; then Algorithms 10-12 reduce to Algorithms 4.1-4.3 of Agarwal and Verma [9], respectively. 
Now, we provide the main results concerning problem (1) with respect to Algorithms 10-12.

Theorem 14. For $i=1,2, \ldots, m$, let $\eta_{i}: H_{i} \times H_{i} \rightarrow H_{i}$ be $\tau_{i}$-Lipschitz continuous operator, let $A_{i}: H_{i} \rightarrow H_{i}$ be $\beta_{i^{-}}$ Lipschitz continuous and $\left(r_{i}, \eta_{i}\right)$-strongly monotone operator, and let $M_{i}: H_{i} \rightarrow 2^{H_{i}}$ be $\left(A_{i}, \eta_{i}\right)$-maximal relaxed monotone operator. Suppose that $U_{i j}: H_{j} \rightarrow C\left(H_{j}\right)$ is $D_{j}-\gamma_{i j}$-Lipschitz continuous for $j=1,2, \ldots, m$ and $j \neq i$ and nonlinear operator $F_{i}: H_{1} \times H_{2} \times \cdots \times H_{m} \rightarrow H_{i}$ is $\left(c_{i}, \mu_{i}\right)$-relaxed cocoercive with respect to $A_{i}$ in the ith argument and $\zeta_{i j}$-Lipschitz continuous in the $j$ th argument for $j=1,2, \ldots, m$. If there exists constant $\rho_{i}>0$, for $i=1,2, \ldots, m$, such that

$$
\begin{gathered}
\frac{\tau_{j}}{r_{j}-\rho_{j} m_{j}} \cdot \sqrt{\beta_{j}^{2}-2 \rho_{j} \mu_{j}+2 \rho_{j} c_{j} \zeta_{j j}^{2}+\rho_{j}^{2} \zeta_{j j}^{2}} \\
\quad+\sum_{i=1, i \neq j}^{m} \frac{\rho_{i} \tau_{i} \zeta_{i j} \gamma_{i j}}{r_{i}-\rho_{i} m_{i}}<1,
\end{gathered}
$$

where $r_{j}-\rho_{j} m_{j}>0$ for $j=1,2, \ldots, m$, then the problem (1) admits a solution $\left(x_{1}^{*}, x_{2}^{*}, \ldots, x_{m}^{*}, u_{12}, \ldots\right.$, $u_{1 m}, \ldots, u_{m 1}, \ldots, u_{m m-1}$ ) (in short, $\left.(*)\right)$, where $x_{i}^{*} \in H_{i}$ and $u_{i j} \in U_{i j}\left(x_{j}^{*}\right)$, for any $i, j=1,2, \ldots, m, j \neq i$, and sequences $\left\{x_{j}^{n}\right\}$ and $\left\{u_{i j}^{n}\right\}$ generated by Algorithm 10 strongly converge to $x_{j}^{*}$ and $u_{i j}(i, j=1,2, \ldots, m, j \neq i)$, respectively.

Proof. For $i=1,2, \ldots, m$, applying Algorithm 10 and Lemma 6 , we have

$$
\begin{aligned}
& \left\|x_{i}^{n+1}-x_{i}^{n}\right\| \\
& \leq\left(1-\lambda_{n}-\delta_{n}\right)\left\|x_{i}^{n}-x_{i}^{n-1}\right\| \\
& +\lambda_{n} \| R_{M_{i}, \rho_{i}}^{A_{i}, \eta_{i}}\left[A_{i}\left(x_{i}^{n}\right)\right. \\
& \left.-\rho_{i} F_{i}\left(u_{i 1}^{n}, \ldots, u_{i i-1}^{n}, x_{i}^{n}, u_{i i+1}^{n}, \ldots, u_{i m}^{n}\right)\right] \\
& -R_{M_{i}, \rho_{i}}^{A_{i}, \eta_{i}}\left[A_{i}\left(x_{i}^{n-1}\right)\right. \\
& \left.-\rho_{i} F_{i}\left(u_{i 1}^{n-1}, \ldots, u_{i i-1}^{n-1}, x_{i}^{n-1}, u_{i i+1}^{n-1}, \ldots, u_{i m}^{n-1}\right)\right] \| \\
& \leq\left(1-\lambda_{n}\right)\left\|x_{i}^{n}-x_{i}^{n-1}\right\| \\
& +\frac{\lambda_{n} \tau_{i}}{r_{i}-\rho_{i} m_{i}} \| A_{i}\left(x_{i}^{n}\right)-A_{i}\left(x_{i}^{n-1}\right) \\
& -\rho_{i}\left[F_{i}\left(u_{i 1}^{n}, \ldots, u_{i i-1}^{n}, x_{i}^{n}, u_{i i+1}^{n}, \ldots, u_{i m}^{n}\right)\right. \\
& -F_{i}\left(u_{i 1}^{n}, \ldots, u_{i i-1}^{n}, x_{i}^{n-1}, u_{i i+1}^{n}, \ldots, u_{i m}^{n}\right) \| \\
& +\frac{\lambda_{n} \tau_{i} \rho_{i}}{r_{i}-\rho_{i} m_{i}} \| F_{i}\left(u_{i 1}^{n}, \ldots, u_{i i-1}^{n}, x_{i}^{n-1}, u_{i i+1}^{n}, \ldots, u_{i m}^{n}\right) \\
& -F_{i}\left(u_{i 1}^{n-1}, \ldots, u_{i i-1}^{n-1}, x_{i}^{n-1}, u_{i i+1}^{n-1}, \ldots, u_{i m}^{n-1}\right) \| .
\end{aligned}
$$

Since $A_{i}$ is $\beta_{i}$-Lipschitz continuous, $F_{i}$ is $\left(c_{i}, \mu_{i}\right)$-relaxed cocoercive with respect to $A_{i}$ in the $i$ th argument, and $F_{i}$ is $\zeta_{i j^{-}}$ Lipschitz continuous in the $j$-th argument for $j=1,2, \ldots, m$, then we have

$$
\begin{aligned}
& \| A_{i}\left(x_{i}^{n}\right)-A_{i}\left(x_{i}^{n-1}\right) \\
& -\rho_{i}\left[F_{i}\left(u_{i 1}^{n}, \ldots, u_{i i-1}^{n}, x_{i}^{n}, u_{i i+1}^{n}, \ldots, u_{i m}^{n}\right)\right. \\
& \left.-F_{i}\left(u_{i 1}^{n}, \ldots, u_{i i-1}^{n}, x_{i}^{n-1}, u_{i i+1}^{n}, \ldots, u_{i m}^{n}\right)\right] \|^{2} \\
& =\left\|A_{i}\left(x_{i}^{n}\right)-A_{i}\left(x_{i}^{n-1}\right)\right\|^{2} \\
& -2 \rho_{i}\left\langle F_{i}\left(u_{i 1}^{n}, \ldots, u_{i i-1}^{n}, x_{i}^{n}, u_{i i+1}^{n}, \ldots, u_{i m}^{n}\right)\right. \\
& -F_{i}\left(u_{i 1}^{n}, \ldots, u_{i i-1}^{n}, x_{i}^{n-1}, u_{i i+1}^{n}, \ldots, u_{i m}^{n}\right) \text {, } \\
& \left.A_{i}\left(x_{i}^{n}\right)-A_{i}\left(x_{i}^{n-1}\right)\right\rangle \\
& +\rho_{i}^{2} \| F_{i}\left(u_{i 1}^{n}, \ldots, u_{i i-1}^{n}, x_{i}^{n}, u_{i i+1}^{n}, \ldots, u_{i m}^{n}\right) \\
& -F_{i}\left(u_{i 1}^{n}, \ldots, u_{i i-1}^{n}, x_{i}^{n-1}, u_{i i+1}^{n}, \ldots, u_{i m}^{n}\right) \|^{2} \\
& \leq \beta_{i}^{2}\left\|x_{i}^{n}-x_{i}^{n-1}\right\|^{2} \\
& -2 \rho_{i}\left[\left(-c_{i}\right) \| F_{i}\left(u_{i 1}^{n}, \ldots, u_{i i-1}^{n}, x_{i}^{n}, u_{i i+1}^{n}, \ldots, u_{i m}^{n}\right)\right. \\
& -F_{i}\left(u_{i 1}^{n}, \ldots, u_{i i-1}^{n}, x_{i}^{n-1}, u_{i i+1}^{n}, \ldots, u_{i m}^{n}\right) \|^{2} \\
& \left.+\mu_{i}\left\|x_{i}^{n}-x_{i}^{n-1}\right\|^{2}\right]+\rho_{i}^{2} \zeta_{i i}^{2}\left\|x_{i}^{n}-x_{i}^{n-1}\right\|^{2} \\
& \leq\left(\beta_{i}^{2}-2 \rho_{i} \mu_{i}+2 \rho_{i} c_{i} \zeta_{i i}^{2}+\rho_{i}^{2} \zeta_{i i}^{2}\right)\left\|x_{i}^{n}-x_{i}^{n-1}\right\|^{2} \text {. }
\end{aligned}
$$

By $D_{j}-\gamma_{i j}$-Lipschitz continuity of $U_{i j}$ and (26), we get

$$
\begin{aligned}
& \| F_{i}\left(u_{i 1}^{n}, \ldots, u_{i i-1}^{n}, x_{i}^{n-1}, u_{i i+1}^{n}, \ldots, u_{i m}^{n}\right) \\
& -F_{i}\left(u_{i 1}^{n-1}, \ldots, u_{i i-1}^{n-1}, x_{i}^{n-1}, u_{i i+1}^{n-1}, \ldots, u_{i m}^{n-1}\right) \| \\
& \leq \| F_{i}\left(u_{i 1}^{n}, u_{i 2}^{n}, \ldots, u_{i i-1}^{n}, x_{i}^{n-1}, u_{i i+1}^{n}, \ldots, u_{i m}^{n}\right) \\
& \quad-F_{i}\left(u_{i 1}^{n-1}, u_{i 2}^{n}, \ldots, u_{i i-1}^{n}, x_{i}^{n-1}, u_{i i+1}^{n}, \ldots, u_{i m}^{n}\right) \| \\
& +\cdots+\| F_{i}\left(u_{i 1}^{n-1}, u_{i 2}^{n-1}, \ldots, u_{i i-1}^{n}, x_{i}^{n-1}, u_{i i+1}^{n}, \ldots, u_{i m}^{n}\right) \\
& \quad-F_{i}\left(u_{i 1}^{n-1}, u_{i 2}^{n-1}, \ldots, u_{i i-1}^{n-1}, x_{i}^{n-1}, u_{i i+1}^{n}, \ldots, u_{i m}^{n}\right) \| \\
& \quad+\| F_{i}\left(u_{i 1}^{n-1}, u_{i 2}^{n-1}, \ldots, u_{i i-1}^{n-1}, x_{i}^{n-1}, u_{i i+1}^{n}, \ldots, u_{i m}^{n}\right) \\
& \quad-F_{i}\left(u_{i 1}^{n-1}, u_{i 2}^{n-1}, \ldots, u_{i i-1}^{n-1}, x_{i}^{n-1}, u_{i i+1}^{n-1}, \ldots, u_{i m}^{n}\right) \| \\
& +\cdots+\| F_{i}\left(u_{i 1}^{n-1}, u_{i 2}^{n-1}, \ldots, u_{i i-1}^{n-1}, x_{i}^{n-1}, u_{i i+1}^{n-1}, \ldots, u_{i m}^{n}\right) \\
& \quad-F_{i}\left(u_{i 1}^{n-1}, u_{i 2}^{n-1}, \ldots, u_{i i-1}^{n-1}, x_{i}^{n-1}, u_{i i+1}^{n-1}, \ldots, u_{i m}^{n-1}\right) \|
\end{aligned}
$$




$$
\begin{aligned}
\leq & \zeta_{i 1}\left\|u_{i 1}^{n}-u_{i 1}^{n-1}\right\|+\cdots+\zeta_{i i-1}\left\|u_{i i-1}^{n}-u_{i i-1}^{n-1}\right\| \\
& +\zeta_{i i+1}\left\|u_{i i+1}^{n}-u_{i i+1}^{n-1}\right\|+\cdots+\zeta_{i m}\left\|u_{i m}^{n}-u_{i m}^{n-1}\right\| \\
= & \sum_{j=1, j \neq i}^{m} \zeta_{i j}\left\|u_{i j}^{n}-u_{i j}^{n-1}\right\| \\
\leq & \sum_{j=1, j \neq i}^{m} \zeta_{i j}\left(1+\frac{1}{n}\right) D_{j}\left(U_{i j}\left(x_{j}^{n}\right), U_{i j}\left(x_{j}^{n-1}\right)\right) \\
\leq & \left(1+\frac{1}{n}\right) \sum_{j=1, j \neq i}^{m} \zeta_{i j} \gamma_{i j}\left\|x_{j}^{n}-x_{j}^{n-1}\right\| .
\end{aligned}
$$

It follows from (32)-(34) that

$$
\begin{aligned}
& \left\|x_{i}^{n+1}-x_{i}^{n}\right\| \\
& \leq\left(1-\lambda_{n}\right)\left\|x_{i}^{n}-x_{i}^{n-1}\right\| \\
& +\frac{\lambda_{n} \tau_{i}}{r_{i}-\rho_{i} m_{i}}\left[\sqrt{\beta_{i}^{2}-2 \rho_{i} \mu_{i}+2 \rho_{i} c_{i} \zeta_{i i}^{2}+\rho_{i}^{2} \zeta_{i i}^{2}}\left\|x_{i}^{n}-x_{i}^{n-1}\right\|\right. \\
& \left.+\left(1+\frac{1}{n}\right) \rho_{i} \sum_{j=1, j \neq i}^{m} \zeta_{i j} \gamma_{i j}\left\|x_{j}^{n}-x_{j}^{n-1}\right\|\right],
\end{aligned}
$$

which implies that

$$
\begin{aligned}
& \sum_{j=1}^{m}\left\|x_{j}^{n+1}-x_{j}^{n}\right\|=\sum_{i=1}^{m}\left\|x_{i}^{n+1}-x_{i}^{n}\right\| \\
& \leq \sum_{i=1}^{m}\left[\left(1-\lambda_{n}\right)\left\|x_{i}^{n}-x_{i}^{n-1}\right\|\right. \\
& +\frac{\lambda_{n} \tau_{i}}{r_{i}-\rho_{i} m_{i}}\left(\sqrt{\beta_{i}^{2}-2 \rho_{i} \mu_{i}+2 \rho_{i} c_{i} \zeta_{i i}^{2}+\rho_{i}^{2} \zeta_{i i}^{2}}\right. \\
& \times\left\|x_{i}^{n}-x_{i}^{n-1}\right\|+\left(1+\frac{1}{n}\right) \rho_{i} \\
& \left.\left.\times \sum_{j=1, j \neq i}^{m} \zeta_{i j} \gamma_{i j}\left\|x_{j}^{n}-x_{j}^{n-1}\right\|\right)\right] \\
& =\sum_{i=1}^{m}\left[\left(1-\lambda_{n}\right)+\frac{\lambda_{n} \tau_{i}}{r_{i}-\rho_{i} m_{i}}\right. \\
& \left.\times \sqrt{\beta_{i}^{2}-2 \rho_{i} \mu_{i}+2 \rho_{i} c_{i} \zeta_{i i}^{2}+\rho_{i}^{2} \zeta_{i i}^{2}}\right]\left\|x_{i}^{n}-x_{i}^{n-1}\right\| \\
& +\left(1+\frac{1}{n}\right) \lambda_{n} \sum_{i=1}^{m} \sum_{j=1, j \neq i}^{m} \frac{\rho_{i} \tau_{i} \zeta_{i j} \gamma_{i j}}{r_{i}-\rho_{i} m_{i}}\left\|x_{j}^{n}-x_{j}^{n-1}\right\| \\
& =\sum_{j=1}^{m}\left[\left(1-\lambda_{n}\right)+\frac{\lambda_{n} \tau_{j}}{r_{j}-\rho_{j} m_{j}}\right.
\end{aligned}
$$

$$
\begin{aligned}
&\left.\times \sqrt{\beta_{j}^{2}-2 \rho_{j} \mu_{j}+2 \rho_{j} c_{j} \zeta_{j j}^{2}+\rho_{j}^{2} \zeta_{j j}^{2}}\right]\left\|x_{j}^{n}-x_{j}^{n-1}\right\| \\
&+\left(1+\frac{1}{n}\right) \lambda_{n} \sum_{j=1}^{m} \sum_{i=1, i \neq j}^{m} \frac{\rho_{i} \tau_{i} \zeta_{i j} \gamma_{i j}}{r_{i}-\rho_{i} m_{i}}\left\|x_{j}^{n}-x_{j}^{n-1}\right\| \\
&=\sum_{j=1}^{m}\left[\left(1-\lambda_{n}\right)+\lambda_{n}\right. \\
& \quad \times\left(\frac{\tau_{j}}{r_{j}-\rho_{j} m_{j}} \sqrt{\beta_{j}^{2}-2 \rho_{j} \mu_{j}+2 \rho_{j} c_{j} \zeta_{j j}^{2}+\rho_{j}^{2} \zeta_{j j}^{2}}\right. \\
&\left.\left.\quad+\left(1+\frac{1}{n}\right) \sum_{i=1, i \neq j}^{m} \frac{\rho_{i} \tau_{i} \zeta_{i j} \gamma_{i j}}{r_{i}-\rho_{i} m_{i}}\right)\right]\left\|x_{j}^{n}-x_{j}^{n-1}\right\| \\
&=\sum_{j=1}^{m}\left[\left(1-\lambda_{n}\right)+\lambda_{n} \theta_{j}^{n}\right]\left\|x_{j}^{n}-x_{j}^{n-1}\right\| \\
& \leq \sum_{j=1}^{m}\left[(1-\omega)+\omega \theta_{j}^{n}\right]\left\|x_{j}^{n}-x_{j}^{n-1}\right\| \\
& f_{j=1}^{m}\left\|x_{j}^{n}-x_{j}^{n-1}\right\|,
\end{aligned}
$$

where

$$
\begin{aligned}
& \omega=\lim _{n \geq 0} \inf \lambda_{n}>0, \quad f_{n}(\omega)=\max _{1 \leq j \leq m}\left\{(1-\omega)+\omega \theta_{j}^{n}\right\}, \\
& \theta_{j}^{n}=\frac{\tau_{j}}{r_{j}-\rho_{j} m_{j}} \sqrt{\beta_{j}^{2}-2 \rho_{j} \mu_{j}+2 \rho_{j} c_{j} \zeta_{j j}^{2}+\rho_{j}^{2} \zeta_{j j}^{2}} \\
& +\left(1+\frac{1}{n}\right) \sum_{i=1, i \neq j}^{m} \frac{\rho_{i} \tau_{i} \zeta_{i j} \gamma_{i j}-\rho_{i} m_{i}}{r_{i}} .
\end{aligned}
$$

By condition (31), we know sequence $\left\{\theta_{j}^{n}\right\}$ is monotonely decreasing and $\theta_{j}^{n} \rightarrow \theta_{j}$ as $n \rightarrow \infty$. Therefore,

$$
f(\omega)=\lim _{n \rightarrow \infty} f_{n}(\omega)=\max _{1 \leq j \leq m}\left\{(1-\omega)+\omega \theta_{j}\right\} .
$$

Since $0<\theta_{j}<1$, for $j=1,2, \ldots, m$, we get $\theta=$ $\max _{1 \leq j \leq m}\left\{\theta_{j}\right\} \in(0,1)$. By Lemma 8, we have $f(\omega)=1-$ $\omega+\omega \theta \in(0,1)$. From (36), it follows that $\left\{x_{j}^{n}\right\}$ are Cauchy sequences and there exists $x_{j}^{*} \in H_{j}$ such that $x_{j}^{n} \rightarrow x_{j}^{*}$ as $n \rightarrow \infty$, for $j=1,2, \ldots, m$.

Next, we show that $u_{i j}^{n} \rightarrow u_{i j} \in U_{i j}\left(x_{j}^{*}\right)$ as $n \rightarrow \infty$ for $i, j=1,2, \ldots, m$ and $j \neq i$. 
It follows from (34) that $\left\{u_{i j}^{n}\right\}$ are also Cauchy sequences. Hence, there exists $u_{i j} \in H_{j}$ such that $u_{i j}^{n} \rightarrow u_{i j}$ as $n \rightarrow \infty$, for $i, j=1,2, \ldots, m, j \neq i$. Furthermore,

$$
\begin{aligned}
& d\left(u_{i j}, U_{i j}\left(x_{j}^{*}\right)\right) \\
& \quad=\inf \left\{\left\|u_{i j}-t\right\|: t \in U_{i j}\left(x_{j}^{*}\right)\right\} \\
& \quad \leq\left\|u_{i j}-u_{i j}^{n}\right\|+d\left(u_{i j}^{n}, U_{i j}\left(x_{j}^{*}\right)\right) \\
& \quad \leq\left\|u_{i j}-u_{i j}^{n}\right\|+D_{j}\left(U_{i j}\left(x_{j}^{n}\right), U_{i j}\left(x_{j}^{*}\right)\right) \\
& \quad \leq\left\|u_{i j}-u_{i j}^{n}\right\|+\gamma_{i j}\left\|x_{j}^{n}-x_{j}^{*}\right\| \rightarrow 0 \quad(n \rightarrow \infty) .
\end{aligned}
$$

Since $U_{i j}\left(x_{j}^{*}\right)$ is closed, we have $u_{i j} \in U_{i j}\left(x_{j}^{*}\right)$, for $j=$ $1,2, \ldots, m, j \neq i$.

Using continuity, $(*)$ that is, $\left(x_{1}^{*}, x_{2}^{*}, \ldots, x_{m}^{*}\right) \in H_{1} \times H_{2} \times$ $\cdots \times H_{m}$ and $u_{i j} \in U_{i j}\left(x_{j}^{*}\right)(i, j=1,2, \ldots, m, j \neq i)$ satisfy (22) and so, in light of Lemma $9,(*)$, is a solution to problem (1). This completes the proof.

Remark 15. If $m=2$, then Theorem 14 reduces to Theorem 4.2 in [9].

From Theorem 14, we have the following results.

Corollary 16. For $i, j=1,2, \ldots, m$ and $j \neq i$, assume that $\eta_{i}$, $A_{i}, M_{i}, U_{i j}$, and $H_{i}$ are the same as in Theorem 14. Let $F_{i}$ : $H_{1} \times H_{2} \times \cdots \times H_{m} \rightarrow H_{i}$ be $\mu_{i}$-strongly monotone with respect to $A_{i}$ in the ith argument and $\zeta_{i j}$-Lipschitz continuous in the $j$ th argument for $i, j=1,2, \ldots, m$ and $j \neq i$. If there exist constants $\rho_{i}>0$, for $i=1,2, \ldots, m$, such that

$$
\frac{\tau_{j}}{r_{j}-\rho_{j} m_{j}} \cdot \sqrt{\beta_{j}^{2}-2 \rho_{j} \mu_{j}+\rho_{j}^{2} \zeta_{j j}^{2}}+\sum_{i=1, i \neq j}^{m} \frac{\rho_{i} \tau_{i} \zeta_{i j} \gamma_{i j}}{r_{i}-\rho_{i} m_{i}}<1
$$

where $r_{j}-\rho_{j} m_{j}>0$, for $j=1,2, \ldots, m$, then problem (1) admits a solution $\left(x_{1}^{*}, x_{2}^{*}, \ldots, x_{m}^{*}, u_{12}, \ldots, u_{1 m}, \ldots, u_{m 1}\right.$, $\left.\ldots, u_{m m-1}\right)$, where, for any $i, j=1,2, \ldots, m, j \neq i, x_{i}^{*} \in H_{i}$ and $u_{i j} \in U_{i j}\left(x_{j}^{*}\right)$ and sequences $\left\{x_{j}^{n}\right\},\left\{u_{i j}^{n}\right\}$ generated by Algorithm 11 strongly converge to $x_{j}^{*}$ and $u_{i j}$, respectively.

Corollary 17. For $i, j=1,2, \ldots, m$ and $j \neq i$, let $\eta_{i}, A_{i}$, $M_{i}, F_{i}$, and $H_{i}$ be the same as in Corollary 16 and let $U_{i j}$ : $H_{j} \rightarrow C B\left(H_{j}\right)$ be $\widehat{H}-\gamma_{i j}$-Lipschitz continuous. If condition (40) in Corollary 16 holds, then problem (1) admits a solution $\left(x_{1}^{*}, x_{2}^{*}, \ldots, x_{m}^{*}, u_{12}, \ldots, u_{1 m}, \ldots, u_{m 1}, \ldots, u_{m m-1}\right)$, where $x_{i}^{*} \in$ $H_{i}$ and $u_{i j} \in U_{i j}\left(x_{j}^{*}\right)$, and sequences $\left\{x_{j}^{n}\right\},\left\{u_{i j}^{n}\right\}(i, j=$ $1,2, \ldots, m, j \neq i)$ generated by Algorithm 12 strongly converge to $x_{j}^{*}$ and $u_{i j}$, for any $i, j=1,2, \ldots, m, j \neq i$, respectively.

\section{Conflict of Interests}

The authors declare that there is no conflict of interests regarding the publication of this paper.

\section{Acknowledgments}

This work was supported by the Cultivation Project of Sichuan University of Science \& Engineering (2011PY01) and the Open Research Fund of Artificial Intelligence Key Laboratory of Sichuan Province (2012RYY04).

\section{References}

[1] Y.-P. Fang, N.-J. Huang, and H.-B. Thompson, "A new system of variational inclusions with $(H, \eta)$-monotone operators in Hilbert spaces," Computers \& Mathematics with Applications, vol. 49, no. 2-3, pp. 365-374, 2005.

[2] W.-Y. Yan, Y.-P. Fang, and N.-J. Huang, "A new system of set-valued variational inclusions with $H$-monotone operators," Mathematical Inequalities \& Applications, vol. 8, no. 3, pp. 537546, 2005.

[3] Y.-P. Fang and N.-J. Huang, "H-monotone operators and system of variational inclusions," Communications on Applied Nonlinear Analysis, vol. 11, no. 1, pp. 93-101, 2004.

[4] H.-W. Cao, "Sensitivity analysis for a system of generalized nonlinear mixed quasi variational inclusions with $H$-monotone operators," Journal of Applied Mathematics, vol. 2011, Article ID 921835, 15 pages, 2011.

[5] H.-Y. Lan, J.-H. Kim, and Y.-J. Cho, "On a new system of nonlinear $A$-monotone multivalued variational inclusions," Journal of Mathematical Analysis and Applications, vol. 327, no. 1, pp. 481-493, 2007.

[6] H.-Y. Lan, "A class of nonlinear $(A, \eta)$-monotone operator inclusion problems with relaxed cocoercive mappings," Advances in Nonlinear Variational Inequalities, vol. 9, no. 2, pp. $1-11,2006$.

[7] J.-W. Peng and L.-J. Zhao, "General system of $A$-monotone nonlinear variational inclusions problems with applications," Journal of Inequalities and Applications, vol. 2009, Article ID 364615, 13 pages, 2009.

[8] R.-U. Verma, "A-monotonicity and applications to nonlinear variational inclusion problems," Journal of Applied Mathematics and Stochastic Analysis, vol. 17, no. 2, pp. 193-195, 2004.

[9] R.-P. Agarwal and R.-U. Verma, "General system of $(A, \eta)$ maximal relaxed monotone variational inclusion problems based on generalized hybrid algorithms," Communications in Nonlinear Science and Numerical Simulation, vol. 15, no. 2, pp. 238-251, 2010.

[10] R.-U. Verma, "Sensitivity analysis for generalized strongly monotone variational inclusions based on the $(A, \eta)$-resolvent operator technique," Applied Mathematics Letters, vol. 19, no. 12, pp. 1409-1413, 2006.

[11] X.-P. Ding and C.-L. Luo, "Perturbed proximal point algorithms for general quasi-variational-like inclusions," Journal of Computational and Applied Mathematics, vol. 113, no. 1-2, pp. 153-165, 2000.

[12] Y.-P. Fang and N.-J. Huang, " $H$-monotone operator and resolvent operator technique for variational inclusions," Applied Mathematics and Computation, vol. 145, no. 2-3, pp. 795-803, 2003.

[13] N.-J. Huang and Y.-P. Fang, "A new class of general variational inclusions involving maximal $\eta$-monotone mappings," Publicationes Mathematicae Debrecen, vol. 62, no. 1-2, pp. 83-98, 2003.

[14] M.-M. Jin, "Generalized nonlinear implicit quasivariational inclusions with relaxed monotone mappings," Advances in Nonlinear Variational Inequalities, vol. 7, no. 2, pp. 173-181, 2004. 
[15] M.-M. Jin, "Iterative algorithm for a new system of nonlinear set-valued variational inclusions involving $(H, \eta)$-monotone mappings," Journal of Inequalities in Pure and Applied Mathematics, vol. 7, no. 2, article 72, 10 pages, 2006.

[16] R.-P. Agarwal, N.-J. Huang, and Y.-J. Cho, "Generalized nonlinear mixed implicit quasi-variational inclusions with set-valued mappings," Journal of Inequalities and Applications, vol. 7, no. 6, pp. 807-828, 2002.

[17] R.-U. Verma, "Approximation solvability of a class of nonlinear set-valued variational inclusions involving $(A, \eta)$-monotone mappings," Journal of Mathematical Analysis and Applications, vol. 337, no. 2, pp. 969-975, 2008.

[18] N.-J. Huang and Y.-P. Fang, "Fixed point theorems and a new system of multivalued generalized order complementarity problems," Positivity, vol. 7, no. 3, pp. 257-265, 2003.

[19] R.-U. Verma, "Projection methods, algorithms, and a new system of nonlinear variational inequalities," Computers \& Mathematics with Applications, vol. 41, no. 7-8, pp. 1025-1031, 2001.

[20] Y.-J. Cho, Y.-P. Fang, N.-J. Huang, and H.-J. Hwang, "Algorithms for systems of nonlinear variational inequalities," Journal of the Korean Mathematical Society, vol. 41, no. 3, pp. 489-499, 2004.

[21] J.-K. Kim and D.-S. Kim, "A new system of generalized nonlinear mixed variational inequalities in Hilbert spaces," Journal of Convex Analysis, vol. 11, no. 1, pp. 235-243, 2004.

[22] R.-U. Verma, "Generalized system for relaxed cocoercive variational inequalities and projection methods," Journal of Optimization Theory and Applications, vol. 121, no. 1, pp. 203-210, 2004.

[23] G. Kassay and J. Kolumbán, "System of multi-valued variational inequalities," Publicationes Mathematicae Debrecen, vol. 56, no. 1-2, pp. 185-195, 2000.

[24] Y. Yao, Y.-C. Liou, C.-L. Li, and H.-T. Lin, "Extended extragradient methods for generalized variational inequalities," Journal of Applied Mathematics, vol. 2012, Article ID 237083, 14 pages, 2012.

[25] M.-A. Noor, K.-I. Noor, Z. Huang, and E. Al-Said, "Implicit schemes for solving extended general nonconvex variational inequalities," Journal of Applied Mathematics, vol. 2012, Article ID 646259, 10 pages, 2012.

[26] S.-B. Nadler Jr., "Multi-valued contraction mappings," Pacific Journal of Mathematics, vol. 30, no. 2, pp. 475-488, 1969. 


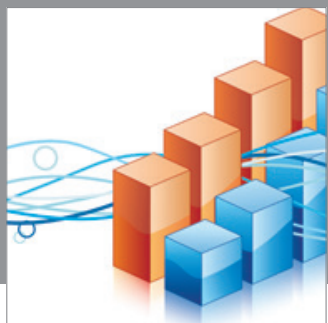

Advances in

Operations Research

mansans

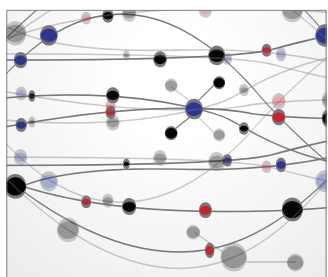

The Scientific World Journal
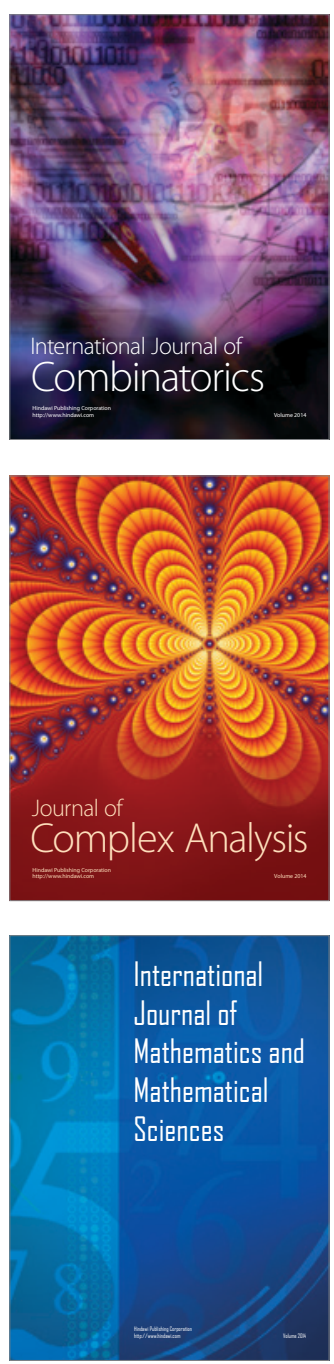
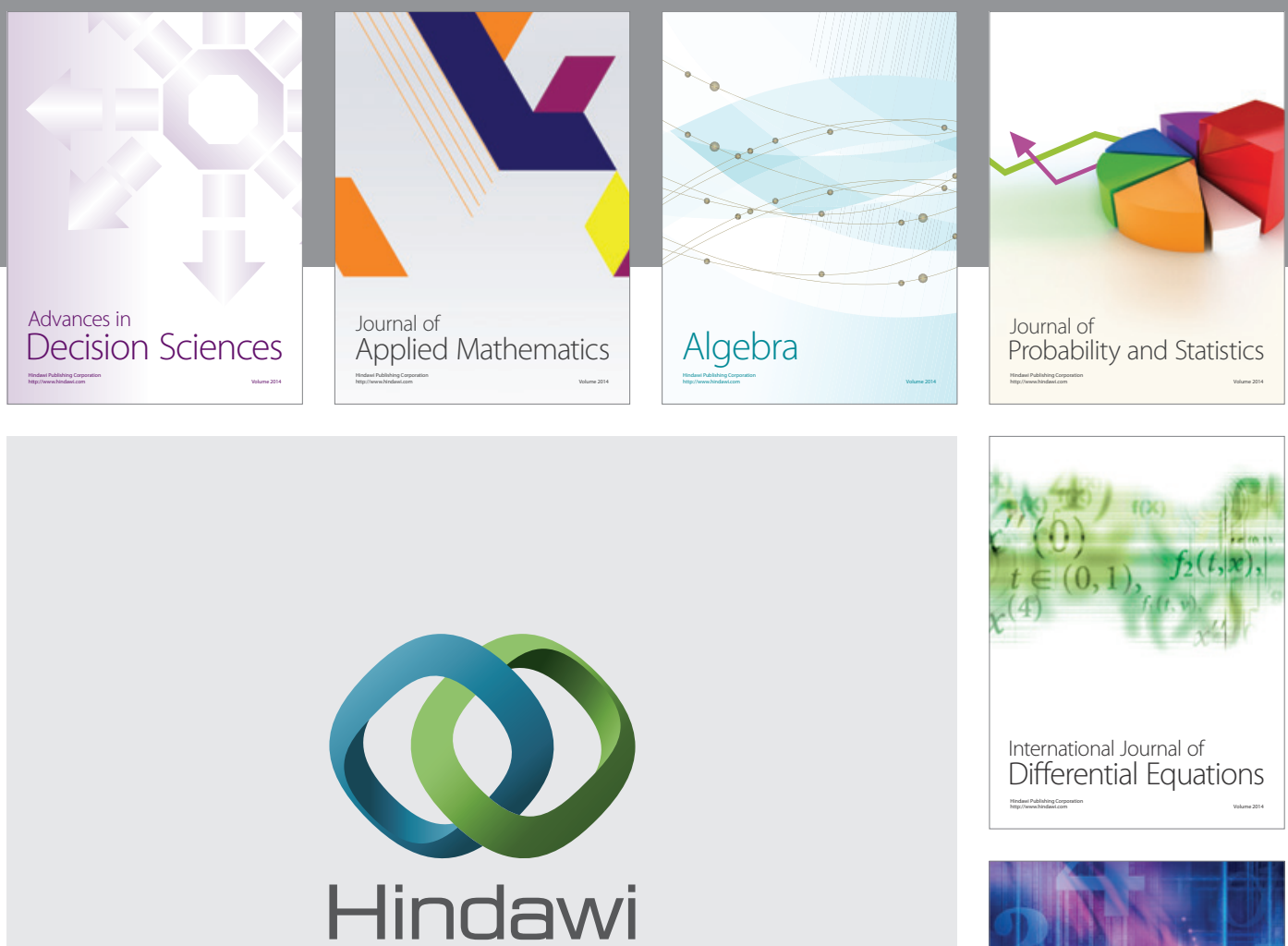

Submit your manuscripts at http://www.hindawi.com
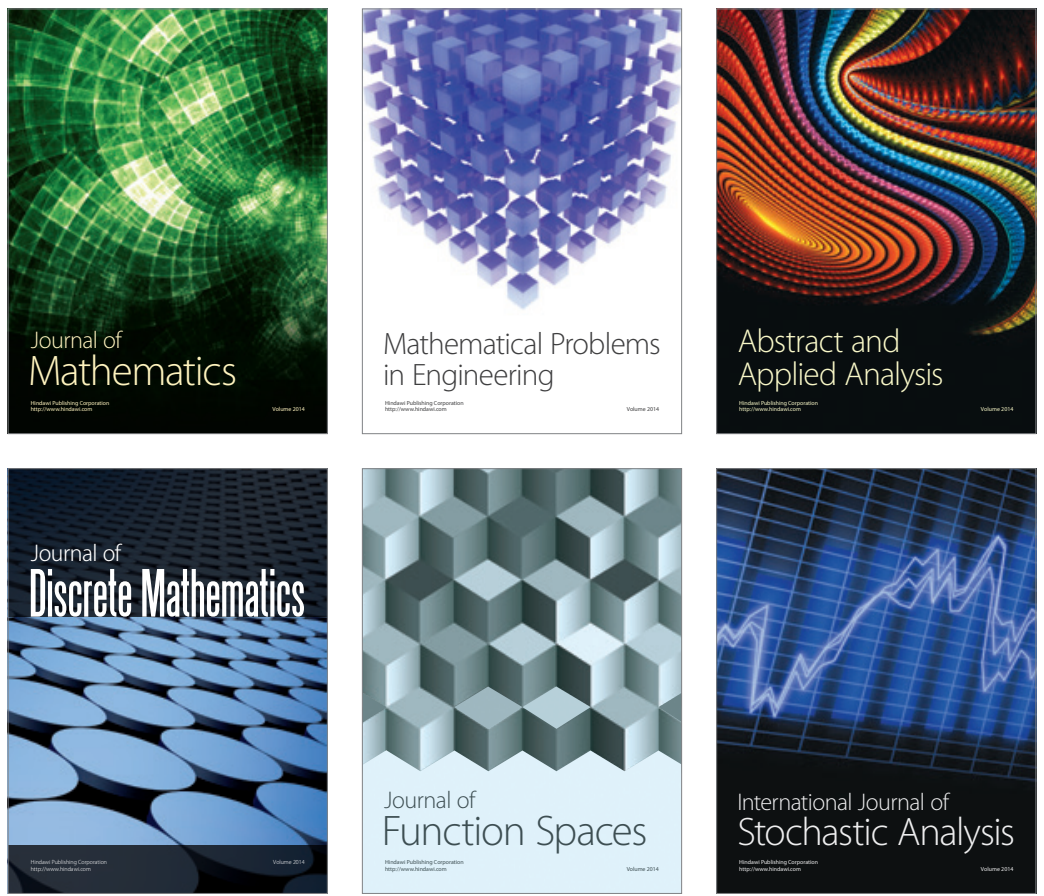

Journal of

Function Spaces

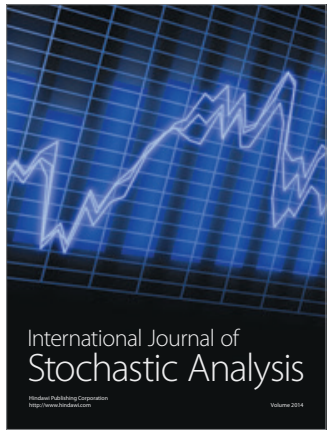

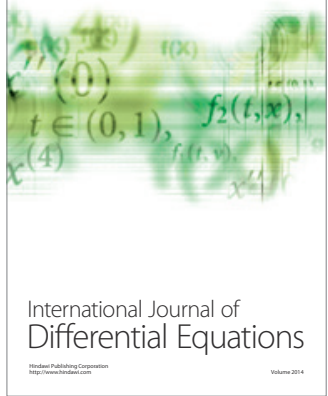
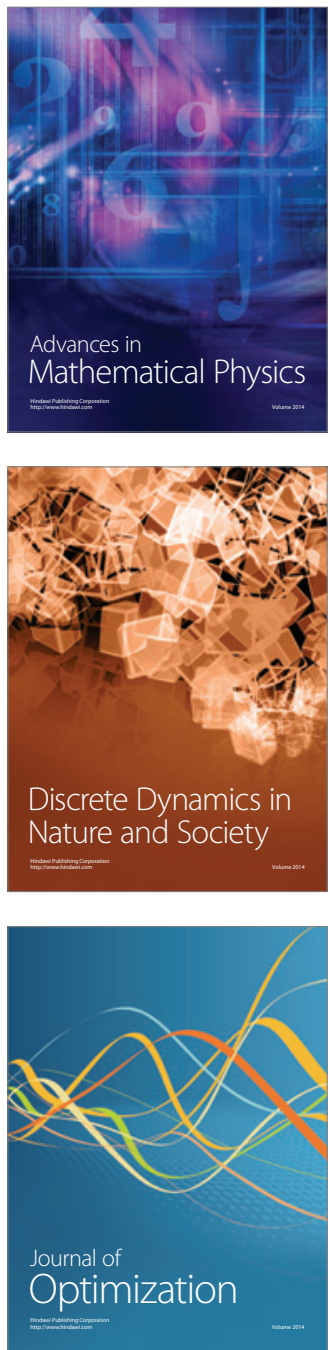